\title{
Colaboración
}

\section{Uso de las herramientas de simulación energética para el estudio del microclima urbano}

Autores: Irina Tumini y Ester Higueras García de la Universidad Politécnica de Madrid

\section{INTRODUCCIÓN}

El microclima urbano juega un rol importante en el consumo energético de los edificios y en las sensaciones de confort en los espacios exteriores. La urgente necesidad de aumentar la eficiencia energética, reducir las emisiones de los contaminantes y paliar la evidente falta de sostenibilidad que afecta a los espacios construidos, ha puesto la atención en el urbanismo bioclimático como referente para una propuesta de cambio en la forma de diseñar y vivir la ciudad ${ }^{1}$. La capacidad de simular el comportamiento térmico del espacio urbano es entonces elemento fundamental en el diseño urbano bioclimático. La introducción del cálculo computarizado ha consentido lograr avances importantes en la modelización y simulación, permitiendo la obtención de resultados cada vez más cercanos al comportamiento real del espacio urbano. A diferencia de los sistemas basados en las observaciones en el espacio real, el uso de los sistemas de simulación aporta algunas ventajas entre las cuales podemos destacar la posibilidad de evaluar escenarios de proyecto, comparando así los resultados en diferentes condiciones ${ }^{2}{ }^{3}$.

Sin embargo las herramientas disponibles tienen características y alcances diferentes por lo que resulta difícil elegir la más oportuna en cada caso. Por eso se ha realizado una revisión crítica de algunas de las herramientas de cálculo existentes, resaltando sus ventajas y limitaciones, proporcionando una información ordenada según una serie de parámetros objetivos que puede ser muy útil para orientar al proyectista entre las diferentes opciones de cálculo.

La selección de las herramientas ha sido enfocada hacía la búsqueda de sistemas que puedan ser empleados por los proyectistas Arquitectos, Urbanistas e Ingenieros, descartando aquellos sistemas que por complejidad, tiempo de ejecución y recursos no son efectivos durante la fase de diseño. Todas tienen un grado de complejidad de manejo medio, aunque en ciertos casos pueda ser necesario un periodo de formación en la herramienta selecciona. Además pueden ejecutarse sin necesitar gran potencia de hardware y, a exclusión de Autodosk Ecotec Analysis 2010, son software libres. Las herramientas analizadas son: SUNTOOL ${ }^{4}$,
SOLENE 5 , RAYMAN ${ }^{6}$, URSOS $^{7}, \mathrm{Au}-$ todosk Ecotec Analysis $2010^{8}$ y Envimet $3.1^{9}$.

Los sistemas evaluados se enfrentan a la estimación del comportamiento urbano utilizando enfoques que implican un cierto grado de aproximación en el acercamiento al cálculo computacional. Los resultados del análisis se ha organizados en tres apartados resumidos a continuación:

Sistema de Cálculo: casi todas las herramientas realizan una simulación de la componente de radiación solar y, exclusión hecha por RayMan, de la transmisión del calor, mientras que solo dos de los programas examinados realizan también una valoración de la dinámica de los fluidos en la microescala. En ambos casos se basan en el cálculo numérico de CFD.

Datos de entrada: todas las herramientas estudiadas permiten la introducción de la geometría 3D, aunque con grandes diferencias en la interfaz utilizada y los datos pedidos. En relación los datos climáticos algunas utilizan datos procedentes de bases de datos estandarizadas, mientras que otras necesitas valores obtenidos de mediciones in situ añadiendo así la dificultad de tener que realizar una campaña de mediciones in situ.

I Santamouris M, Asimakopoulos D N, Assimakopoulos V D, et al. Energy and Climate in the Urban Built Environment. . SANTAMOURIS, M. ed., Athens, Greece: University of Athens, 2001.410 p. ISBN 978-7-873936-90-7.

2 Ali-Toudert Fazia, Mayer Helmut. "Numerical Study on the Effects of Aspect Ratio and Orientation of an Urban Street Canyon on Outdoor Thermal Comfort in Hot and Dry Climate." Building and Environment, 2, 2006, vol. 41, no. 2. pp. 94-108. D01: 10.1016/i.buildenv.2005.01.013.

3 Mirzaei Parham A., Haghighat Fariborz. "Approaches to Study Urban Heat Island - Abilities and Limitations." Building and Environment, 10, 2010, vol. 45, no. 10. pp. 2192-2201. D0l: 10.1016/i.buildenv.2010.04.001.

4 Robinson D, Campbell N, Gaiser W, et al. "SUNtool - A New Modelling Paradigm for Simulating and Optimising Urban Sustainability." Solar Energy. 2007. vol. 81, no. 9. pp. 1196-1211. D01: 10.1016/i.solener.2007.06.002.

5 http://www.cerma.archi.fr/CERMA/expertise/solene/

6 Matzarakis A., Rutz F.and Mayer, H. "Modelling Radiation Fluxes in Simple and Complex Environments—application of the RayMan Model." International Journal of Biometeorology, 2007, vol. 51 , no. 4. pp. 323-334. D01:10.1016/i.jweia.2012.02.006.

7 http://gee.unizar.es/

8 Marsh A. Autodesk-Ecotect (Version 5.6). , 2010.

9 Bruse M. ENVI-Met Website. Online: Http://Www.Envimet.Com, 2004. 


\section{Colaboración}

Valores de Salida de la Herramienta: en cuanto a los resultados hay muchas diferencias entre las herramientas seleccionadas. La mayoría están enfocadas a la eficiencia energética de los edificios y en el estudio de nuevos desarrollos y sólo dos proporcionan información sobre las condiciones de los espacios abiertos.

El trabajo demuestra que cada sistema es eficaz para algunos objetivos específicos por lo que la realización de un estudio exhaustivo del comportamiento del entorno urbano comporta el uso de diferentes herramientas. Podemos también resaltar que las herramientas que consideran el efecto del movimiento de fluidos emplean modelos más cercanos al comportamiento real, lo que supone a la vez una mayor dificultad de manejo e inversión de tiempo para el cálculo computacional.

En general podemos concluir que las herramientas actualmente dispo- nibles en el mercado emplean métodos simplificados por lo que se deben asumir valores por defectos y limitar su flexibilidad en la introducción de variables para hacer que puedan ser empleadas por usuarios con un grado de especialización medio. Su uso se justifica en una fase de proyecto en orientar las estrategias de diseño y evaluar entre diferentes escenarios el que muestra mejores prestaciones en lugar de buscar estimaciones de valores cercanos a la realidad. Considerar la componente microclimática en un proyecto comporta beneficios a largo plazo, ya que es muy difícil cambiar el comportamiento térmico de una porción de ciudad con una geometría determinada. Las herramientas de simulación permiten realizar una evaluación objetiva de las estrategias de diseño y por eso su uso debería apoyar los proyectistas desde las primeras fases de toma de decisiones.

\section{AGRADECIMIENTOS}

Este trabajo ha sido realizado dentro del marco del proyecto de investigación ECOURBAN - "Metodología para la Evaluación del Impacto Energético y Medioambiental en el Ecodiseño de Urbanizaciones" cofinanciado por el Ministerio de Ciencia e Innovación dentro del Plan Nacional de investigación de I+D+i 2008-2011 - Numero de Referencia ENE2010-19850.

\section{REFERENCIA}

TUMINI I, HIGUERAS-GARCIA E. "ALCANCES Y LIMITACIONES DE LAS HERRAMIENTAS DE SIMULACIÓN PARA EL ESTUDIO DEL MICROCLIMA URBANO" DYNA Energía y Sostenibilidad. ENERO 2013. Vol. 2-1 p.[No Consta]. DOI: http://dx.doi.org/10.6036/ ES6921 